\title{
In vitro modulation of alkaline phosphatase activity of Saccharomyces cerevisiae grown in low or high phosphate medium
}

\author{
J. Fernandes, R. Amorim, I. Azevedo and M.J. Martins \\ Departamento de Bioquímica, Faculdade de Medicina (U38-FCT), Universidade do Porto, Porto, Portugal \\ Correspondence to: M.J. Martins, Departamento de Bioquímica, Faculdade de Medicina (U38-FCT), \\ Universidade do Porto, Alameda Prof. Hernâni Monteiro, 4200-319 Porto, Portugal \\ Fax: +351-22-551-3624. E-mail: mmartins@med.up.pt
}

\begin{abstract}
Our objective was to characterize the modulation of the activity of Saccharomyces cerevisiae alkaline phosphatases (ALPs) by classic inhibitors of ALP activity, cholesterol and steroid hormones, in order to identify catalytic similarities between yeast and mammalian ALPs. S. cerevisiae expresses two ALPs, coded for by the PHO8 and PHO13 genes. The product of the PHO8gene is repressible by $\mathrm{Pi}$ in the medium. ALP activity from yeast (grown in low or high phosphate medium) homogenates was determined with $\mathrm{p}$-nitrophenylphosphate as substrate, $\mathrm{pH} 10.4$ (IPiALP or hPiALP, respectively). Activation of hPiALP was observed with $5 \mathrm{mM}$ L-amino acids (L-homoarginine - 186\%, L-leucine - 155\% and L-phenylalanine - 168\%) and with $1 \mathrm{mM}$ levamisole (122\%; percentage values, in comparison to control, of recovered activity). EDTA (5 mM) and vanadate (1 mM) distinctly inhibited hPiALP (2 and 20\%, respectively). L-homoarginine (5 mM) had a lower activating effect on IPiALP (166\%) and was the strongest hPiALP activator. Corticosterone $(5 \mathrm{mM})$ inhibited hPiALP to $90 \%$, but no effect was observed in low phosphate medium. Cholesterol, ß-estradiol and progesterone also had different effects on IPiALP and hPiALP. A concentrationdependent activation of IPiALP minus hPiALP was evident with all three compounds, most especially with $ß$-estradiol and cholesterol. These results do not allow us to identify similarities of the behavior of $S$. cerevisiae ALPs and any of the mammalian ALPs but allow us to raise the hypothesis of differential regulation of $S$. cerevisiae ALPs by L-homoarginine, $ß$-estradiol and cholesterol and of using these compounds to discriminate between S. cerevisiae IPiALP and hPiALP.
\end{abstract}

Key words: Saccharomyces cerevisiae; Alkaline phosphatase inhibitors; PHO8 and PHO13; Cholesterol; Steroid hormones

A preliminary report of this manuscript was presented in abstract form at the Experimental Biology Meeting, San Francisco, CA, USA, April 1-5, 2006. [Caldeira J, Amorim R, Azevedo I, Martins MJ. Modulation of alkaline phosphatases from Saccharomyces cerevisiae. Faseb J 2006; 20: A51 (Abstract 76.2)].

Research supported by FCT (POCI, FEDER and Programa Comunitário de Apoio).

Received June 9, 2007. Accepted November 5, 2007

\section{INTRODUCTION}

Alkaline phosphatase (ALP; orthophosphoric-monoester phosphohydrolase, alkaline optimum) represents a family of several isoenzymes and isoforms whose expression depends on the species being considered and on physiological/pathological conditions (1-3). ALP substrates (either natural or artificial) possess a phosphate residue $(\mathrm{Pi})$ in a terminal position. Pi released from an ALP substrate molecule is either released into the reaction medium (ALP functioning as phosphohydrolase) or transferred to an appropriate acceptor (ALP functioning as phosphotransferase) (1-8).

The extent of natural ALP substrates and in vivo specific ALP functions are still unknown. ALP participates, along with other enzymes, in Pi homeostasis (in a cyclic pathway of polyphosphate synthesis and degradation), a process including phosphorous acquisition and metabolic integration of $\mathrm{Pi}$ in Saccharomyces cerevisiae, and eutopic or ectopic bone mineralization in mammals (1-3,5-18).

ALP has also been associated with various fundamen- 
tal cellular processes (differentiation, proliferation and cellular signaling, meiosis, mitosis, apoptosis, cell migration, immunological defense, and DNA synthesis regulation) in several species $(1-3,12,15-17)$.

In S. cerevisiae, ALPs are the product of two structural genes: PHO8 and PHO13, both located on chromosome IV. PHO8 ALP (E.C. 3.1.3.1) is an $\mathrm{Mg}^{2+} / \mathrm{Zn}^{2+}$-dependent dimeric protein similar to an ALP found in Escherichia coli and in mammalian cells, is very unspecific in terms of substrates and is located in the cell vacuole. The product of the PHO13gene (E.C. 3.1.3.41) is a monomeric protein, is highly specific for $p$-nitrophenylphosphate ( $p$-NPP) and histidinylphosphate (not attacking other substrates at a significant rate), and, like PHO8 ALP, is also highly dependent on $\mathrm{Mg}^{2+}$. The nonspecific enzyme is repressible by $\mathrm{Pi}$ in the medium, whereas the specific ALP is synthesized constitutively regardless of the $\mathrm{Pi}$ concentration in the medium. The repressible ALP, however, shows a significant level of activity even under repressible conditions. It has been reported that repressible ALP activity, due to transcription of the $\mathrm{PHO} 8 \mathrm{gene}$, is two to three times higher in low than in high Pi medium (5-7,11,19-22). Both $S$. cerevisiae ALPs have protein and/or peptide phosphatase activity (5-7).

The four ALP isoenzyme activities expressed in mammals, i.e., tissue-nonspecific, intestinal, placental, or germcell ALP, with different distributions among species, can be distinguished by their differential inhibition by several compounds such as L-amino acids or levamisole. Unlike mammalian ALPs, bacterial ALPs are not stereospecifically inhibited by L-amino acids through a non-competitive mechanism (1-3,17,23-25).

Since we found no data about the modulation of $S$. cerevisiae ALP activity, in the present study we characterized the modulation of $S$. cerevisiae ALP activity by Lamino acids, cholesterol and steroid hormones. This characterization will show the putatively different catalytic behavior of $\mathrm{PHO} 8$ and $\mathrm{PHO} 13$ ALPs and possibly permit inferences about ALP functions in yeast. Our group has a large experience in comparative modulation of mammalian ALPs and has carried out extensive research on ALP function $(3,13-17,26-28)$. Additionally, by also examining the modulation of $S$. cerevisiae ALP activity by levamisole, EDTA and vanadate, we set out to identify catalytic similarities between yeast and mammalian ALPs in order to find out if $S$. cerevisiae ALPs could be used as a study model of mammalian ALPs.

\section{MATERIAL AND METHODS}

p-NPP (Sigma 104D, St. Louis, MO, USA), p-nitrophenol (p-NPL; Sigma 104-8), bovine serum albumin (BSA) (A-
4503), L-amino acids, levamisole (L-9756), orthovanadate (S-6508), cholesterol (C-8667), and steroid hormones were purchased from Sigma (Sigma Alcobendas, Madrid, Spain). Yeast growth medium was purchased from Difco ${ }^{\mathrm{TM}}$ (YM Broth - 271120, yeast malt, Voigt Global Distribution Inc., Lawrence, KS, USA). All other reagents were of the highest quality and purity available.

\section{Yeast and yeast growth conditions}

S. cerevisiae strain 3507 (wild type) from the Portuguese Yeast Cell Culture (Gulbenkian Institute of Science, Lisbon, Portugal) was kindly provided by ESBUC, College of Biotechnology, Portuguese Catholic University, Porto, Portugal. The strain was grown in 250-mL Schott flasks with $150 \mathrm{~mL}$ medium under slow rotary agitation at $30^{\circ} \mathrm{C}$ on yeast malt medium supplemented with $\mathrm{K}_{2} \mathrm{HPO}_{4}$ at two different final concentrations: $3000 \mathrm{mg} / \mathrm{L}$ (high phosphate medium) and $30 \mathrm{mg} / \mathrm{L}$ (low phosphate medium) (11); the flasks were covered with gauze.

\section{Yeast sample collection}

Yeast samples were collected from the exponential phase. Yeast cells were separated by centrifugation at $6000 \mathrm{~g}$ for $10 \mathrm{~min}$ at $4^{\circ} \mathrm{C}$ and washed once with $5 \mathrm{~mL}$

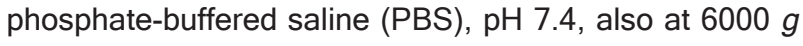
for $10 \mathrm{~min}$ at $4^{\circ} \mathrm{C}$. Then, the yeast pellet was resuspended in $1 \mathrm{~mL}$ PBS and stored at $-80^{\circ} \mathrm{C}$ for no longer than one month.

\section{Alkaline phosphatase activity modulation}

On the day of the experiment, yeast samples were homogenized with a Heidolph homogenizer in PBS + 0.5\% Triton X-100 (4:1) $(13,14,20,22)$ in a final volume of 1.25 $\mathrm{mL}$ and kept on ice. The final concentration of Triton $\mathrm{X}-100$ in the ALP sample incubation medium was lower than its critical micellar concentration. The reaction mixtures (final volume of $500 \mu \mathrm{L}$ ) contained $80 \mathrm{mM}$ Tris, $\mathrm{pH} 10.4,0.4 \mathrm{mM}$ $\mathrm{MgCl}_{2}, 20 \mu \mathrm{L}$ yeast sample from high or low phosphate growth medium, $0.3760 \mathrm{mg}$ of $\mathrm{p}-\mathrm{NPP}$ and $50 \mu \mathrm{L}$ of the compound solution to be tested. The enzymatic reaction was started by adding the ALP substrate. Incubation took place at $37^{\circ} \mathrm{C}$ for $15 \mathrm{~min}$, was stopped by the addition of 2 $\mathrm{mL} 20 \mathrm{mM} \mathrm{NaOH}$ (ice-cold) and then the reaction mixture was placed on ice for $10 \mathrm{~min}$. At the end of this period, a centrifugation was performed at $10,000 \mathrm{~g}$ for $16 \mathrm{~min}$ at $20^{\circ} \mathrm{C}$. The supernatant was collected and the p-NPL released by ALP activity from yeast homogenates (grown in low or high phosphate medium, IPiALP or hPiALP, respectively), in the presence or absence of the compounds to be tested, was quantified by absorbance measurements at 410 nm (Spectronic Genesys 5, Milton Roy, Rochester, 
NY, USA). For both IPiALP and hPiALP there was linearity with incubation time from 5 to $20 \mathrm{~min}$. A p-NPL calibration curve was performed in order to calculate ALP activity. Lamino acids with Sigma numbers given within parentheses (1 and $5 \mathrm{mM}$ : L-phenylalanine (P-2126), L-leucine (L8912), and L-homoarginine (H-1007)), EDTA (1 and 5 $\mathrm{mM})$, vanadate $(0.1$ and $1 \mathrm{mM})$, and levamisole $(0.1$ and 1 $\mathrm{mM}$ ) were dissolved in water. Cholesterol (C-8667), progesterone (P-0130), corticosterone (C-2505), and ß-estradiol (E-8875) (0.01-5 mM) were dissolved in ethanol. Controls for drugs were run in the presence of a corresponding volume of solvent. All assays for each compound at the different concentrations tested were always carried out using the same homogenate batch (either IPiALP or hPiALP homogenates). The effect of each compound tested against ALP activity was determined as a percentage of the corresponding control ALP activity (representing the recovered activity). The buffer $\mathrm{pH}$ value used in the present study was chosen to ensure that only ALP activity would be measured since the ALP molecules were not isolated but their activity was measured in yeast homogenates. All assays were performed in triplicate (differences between replicates were always lower than $5 \%$ ).

\section{Protein determination}

Protein concentration was determined as described by Bradford (29), with BSA as standard. For each yeast growth condition (low or high phosphate medium), the protein concentration of the homogenates used was similar.

\section{Statistical analysis}

Data are reported as means \pm SD and the significance of differences between means was assessed by the paired or unpaired Student $t$-test.

\section{RESULTS}

ALP activity was about $30 \%$ lower in yeast homogenates obtained from S. cerevisiae grown in high phosphate medium than in yeast homogenates grown in low phosphate medium (results not shown).

L-homoarginine, L-leucine and L-phenylalanine significantly activated $\mathrm{hPiALP}$ and IPiALP in a concentrationdependent manner (Figures 1 and 2). For each of the tested concentrations (hPiALP), the results obtained with each L-amino acid were significantly different $(P=0.002$ $0.03)$, except for L-phenylalanine versus L-leucine ( $5 \mathrm{mM})$ and $L$-homoarginine versus the other two L-amino acids ( 1 $\mathrm{mM}$; statistical results not shown in Figure 1). L-homoarginine $(5 \mathrm{mM})$ had a significantly lower activating effect on IPiALP than on hPiALP (statistical result not shown, comparison of Figures 1 and 2).

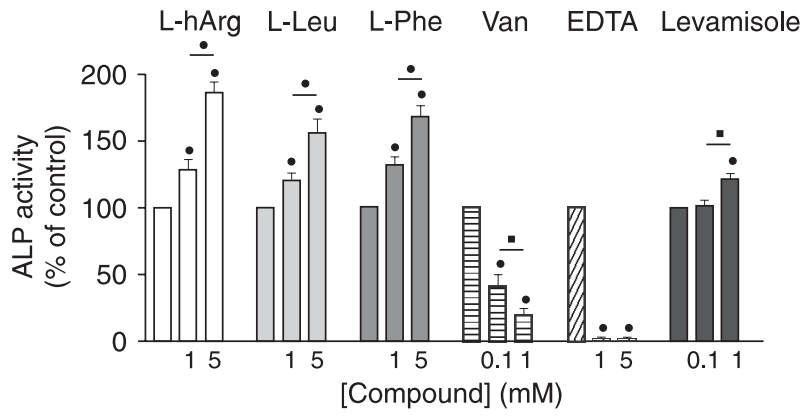

Figure 1. Effect of L-amino acids, EDTA, orthovanadate, and levamisole on alkaline phosphatase (ALP) activity from Saccharomyces cerevisiae homogenates. Yeast was grown in high phosphate medium (3000 mg Pi/L YM medium, $\mathrm{N}=5$ ). Data are reported as arithmetic means \pm SD and as percentage of the corresponding control activity $(=100 \%(173.3 \pm 48.83 \mathrm{nmol} \mathrm{p}$ nitrophenol released $\mathrm{min}^{-1} \mathrm{~mL}$ homogenate ${ }^{-1}$ ); first column of each set of results), determined as described in Material and Methods. Assays were performed in triplicate. Symbols at the top of the columns indicate comparison to the respective control of each compound. Symbols at the top of the horizontal bars indicate the comparison of the two concentrations. Circles indicate $\mathrm{P} \leq 0.005$ and squares indicate $\mathrm{P} \leq 0.05$. Leu = L-leucine; Phe = L-phenylalanine; hArg = L-homoarginine; Van = orthovanadate.

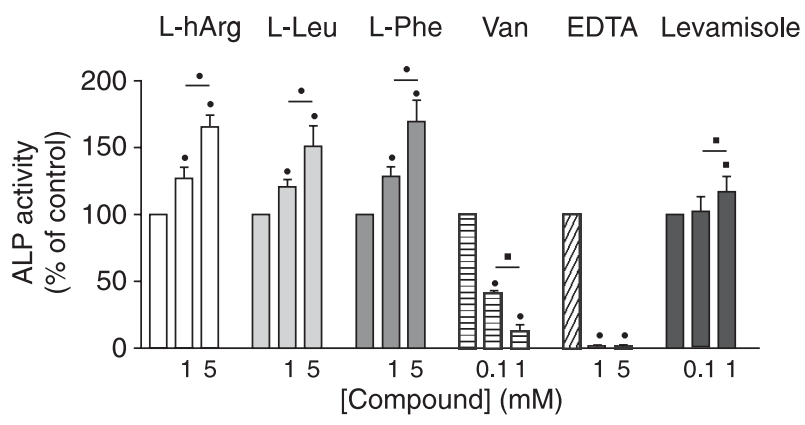

Figure 2. Effect of L-amino acids, EDTA, orthovanadate, and levamisole on alkaline phosphatase (ALP) activity from Saccharomyces cerevisiae homogenates. Yeast was grown in low phosphate medium (30 mg Pi/L YM medium, $\mathrm{N}=7$ ). Data are reported as arithmetic means \pm SD and as percentage of the corresponding control activity $(=100 \%(235.8 \pm 56.33 \mathrm{nmol}$ p-nitrophenol released $\mathrm{min}^{-1} \mathrm{~mL}$ homogenate ${ }^{-1}$ ); first column of each set of results), determined as described in Material and Methods. Assays were performed in triplicate. Symbols at the top of the columns indicate comparison to the respective control of each compound. Symbols at the top of the horizontal bars indicate the comparison of the two concentrations. Circles indicate $P \leq 0.005$ and squares indicate $\mathrm{P} \leq 0.05$. Leu $=\mathrm{L}$-leucine; $\mathrm{Phe}=\mathrm{L}$-phenylalanine; hArg = L-homoarginine; Van = orthovanadate. 
hPiALP and IPIALP were significantly inhibited by both EDTA and vanadate, although with different potencies when comparing both compounds, and in an apparently concentration-independent way for EDTA (Figures 1 and 2 ). These inhibitory effects were significantly different in each growth condition (statistical results not shown in Figures 1 and 2; P < 0.005; low and high EDTA concentrations compared to low and high vanadate concentrations, respectively).

Levamisole $(1 \mathrm{mM})$ significantly activated $\mathrm{hPiALP}$ and IPiALP (Figures 1 and 2). Levamisole effect was significantly different from that of the L-amino acids in each growth condition $(P<0.005$; low and high levamisole concentrations compared to low and high L-amino acid concentrations, respectively, except for IPIALP in levamisole versus L-leucine at the lower concentrations, where $P<0.05$; statistical results not shown in Figures 1 and 2).

Regarding the steroid compounds tested (cholesterol, ß-estradiol, progesterone, and corticosterone), only $5 \mathrm{mM}$ corticosterone had an effect on hPiALP, i.e., significant inhibition (statistical result not shown; $P \leq 0.005$ ). This effect was only significantly different from that of $5 \mathrm{mM}$ progesterone (statistical result not shown; $P=0.002$ ). The inhibition by $5 \mathrm{mM}$ corticosterone observed in high phosphate medium was not observed when yeast was grown in low phosphate medium (result not shown). Cholesterol, ßestradiol and progesterone had different effects on IPiALP and hPiALP activities (statistical results not shown for IPiALP minus hPiALP: significantly different for $5 \mathrm{mM}$ for the first two compounds and for $1 \mathrm{mM}$ for the last one; $\mathrm{P} \leq$ 0.05). Among these three steroid compounds, ß-estradiol presented the strongest concentration-dependent activation of IPiALP minus hPiALP and closely followed by cholesterol (results not shown). Progesterone and corticosterone (1 $\mathrm{mM})$ had significantly different $(P=0.007)$ effects on IPiALP (statistical result not shown) and the same tendency was observed at $5 \mathrm{mM}$ for the same hormones $(P=0.059)$. Progesterone $(1 \mathrm{mM})$ activated IPIALP (statistical result not shown, $\mathrm{P}<0.05)$.

\section{DISCUSSION}

In the present study, the yeast $S$. cerevisiae was grown either in a low or high phosphate medium (30 or 3000 mg/ L) (11). We observed that these distinct growth conditions induced a $30 \%$ difference in total ALP activity in the corresponding $S$. cerevisiae homogenates. This level of reduction of ALP activity induced by $\mathrm{Pi}$ concentration in the growth medium agrees with data from Refs. 11 and 21 .

Mammalian ALPs can be differentially inhibited by Lamino acids, levamisole and EDTA. L-phenylalanine and L-leucine strongly inhibit intestinal, placental and germ cell
ALPs (tissue-specific ALPs). Germ cell ALP is more sensitive to inhibition by L-leucine and EDTA than placental ALP. L-homoarginine strongly inhibits tissue-nonspecific ALP and levamisole inhibits placental and tissue-nonspecific isoenzymes. Levamisole, L-phenylalanine and Lhomoarginine, which permit discrimination between tissue-nonspecific and tissue-specific ALPs, have been used for the clinical quantitation and identification of ALP isoenzymes for almost a century $(2,24,25,30)$. Vanadate is an ALP inhibitor $(12,17,31)$. Corticosterone $(5 \mathrm{mM})$ inhibits rat renal ALP, but not rat liver ALP, and progesterone has no effect on rat renal or liver ALP (13).

To our knowledge, none of the compounds used in this study, with the exception of EDTA, have been tested before in ALPs from $S$. cerevisiae; EDTA has been described to also inhibit Serratia marcescens ALP $(5,7,32)$. The assay conditions used in the present study (ALP sample/homogenate preparation, p-NPP concentration, buffer (type, concentration and $\mathrm{pH}$ value) as well as magnesium concentration) were the same as those we have been using with mammalian ALP homogenate samples $(3,13,14,26,27)$ in order to permit comparison of the results. The concentration ranges for L-amino acids, levamisole, EDTA, vanadate, progesterone, and corticosterone chosen to be tested upon $S$. cerevisiae ALPs in the present study are the same as those tested on mammalian ALPs either in our laboratory or by other authors $(7,12$, 16,24,25).

For L-amino acids, instead of their reported inhibition, we observed activation of ALP activity in both $S$. cerevisiae homogenates. L-homoarginine ( $5 \mathrm{mM})$ was both the strongest activator of hPiALP and the better discriminator between IPIALP and hPiALP. EDTA and vanadate strongly inhibited IPiALP and hPiALP, each compound with the same potency in both types of ALP samples. ALP inhibition by EDTA was not a surprise since ALP needs zinc and magnesium to perform catalysis and EDTA was tested in a concentration range higher than the $\mathrm{Mg}^{2+}$ concentration in the incubation medium. The similar effect of levamisole on either IPiALP or hPiALP was different from that described for tissue-nonspecific ALP. Progesterone and corticosterone acted on hPiALP as described by our group for mammalian (rat) tissue-nonspecific ALP (13). ß-estradiol, progesterone and cholesterol acted similarly on hPiALP and IPiALP minus hPiALP, i.e., they had no significant effect on the former ALP activity and induced a concentration-dependent activation of the latter ALP activity (most especially ß-estradiol and cholesterol).

Under special conditions regarding substrate and magnesium, human ALP activation by L-phenylalanine (in a concentration range similar to that used in the present 
study) has been reported (23). Human ALPs show different susceptibilities to inactivation or activation by $0.01 \mathrm{mM}$ $0.1 \mathrm{M}$ EDTA (30) and calf intestinal ALP is activated by low EDTA concentrations, $20-400 \mu \mathrm{M}$, and irreversibly inhibited by higher concentrations, $1 \mathrm{mM}$ and above (31). Levamisole inhibition of rat liver ALP decreases with increasing levamisole concentration (Martins MJ, unpublished results).

S. cerevisiae is a genetically well-defined organism, easy to manipulate, that grows very fast, its growth and cell division being easily controlled by incubation conditions (33). Thus, S. cerevisiae ALP (either in living cells or in yeast homogenates or extracts) would be most useful as a model to study the modulation of mammalian ALPs. Unfortunately our results did not identify similarities between the behavior of $S$. cerevisiae IPiALP or hPiALP and any of the four mammalian ALPs.

However, our results allow us to raise the hypothesis of differential regulation of $S$. cerevisiae ALPs by L-homoarginine, cholesterol and $ß$-estradiol, all found in yeast metabolism.

In S. cerevisiae, ß-estradiol can be obtained from estrones present in the incubation medium and dehydrogenated by an $S$. cerevisiae peroxisomal multifunctional enzyme that is homologous to a mammalian protein. $S$. cerevisiae possesses a high-affinity estrogen-binding protein (34-36). The effect of this steroid compound on ALP should be further studied. Estrogen has been suggested to cause shortening of the unbudded period of $S$. cerevisiae old cells by stimulating energy metabolism (37). The PHO8 gene of $S$. cerevisiae also codes for the yeast fructose-2,6bisphosphate 6 -phosphatase $(5,38)$. On the other hand, ALP activity has been associated with proliferation and differentiation in several species (1-3,12). A truncated form of PHO8 ALP (lacking 62 amino acids from the N-terminus) obtained from a cell-free extract of $S$. cerevisiae has the ability to convert farnesyl diphosphate into E,E-farnesol (8), suggesting, in association with our results, that this enzyme activity might be involved in cholesterol synthesis regulation.

Modulation of $S$. cerevisiae PHO8 ALP may allow regulation of intracellular levels of nickel, since this enzyme is located in the vacuole where the metal is accumulated (11).

ALP may remove Pi residues from membrane phosphoproteins and from nucleotides such as ATP and AMP and may most probably be involved in transmembrane transport system regulation by modifying the phosphorylation level of transporters or of molecules that regulate them. Supporting this idea is the fact that ALP is an ubiquitous enzyme from humans to Dictyostelium discoi- deum $(1,15-17,20-22)$, located in membranes.

ALP is associated with lipid intestinal transport in mammals, is involved in the modulation of organic cation transport in rat isolated hepatocytes, Caco- 2 and RBE4 cells, as well as in insulin internalization in RBE4 cells (1-3,15$17,39,40)$. It also seems to modulate taurocholate uptake in rat isolated hepatocytes (3).

Although in the present study only the acute effects of ALP modulatory compounds on $S$. cerevisiae homogenates were investigated, the observed differential modulation of S. cerevisiae ALPs might be used to test the hypothesis of yeast transport system regulation by ALP activity since both PHO8 and PHO13 ALPs dephosphorylate phosphoproteins and/or phosphopeptides (5-7).

The present results regarding in vitro IPiALP and hPiALP modulation did not allow the identification of similarities in the behavior of $S$. cerevisiae ALPs and of any of the mammalian ALPs. However, they allow us to raise the hypothesis of differential regulation of $S$. cerevisiae ALPS by L-homoarginine, ß-estradiol and cholesterol and, consequently, to propose the use of these compounds to discriminate between $S$. cerevisiae IPiALP and hPiALP.

\section{ACKNOWLEDGMENTS}

We would like to thank Dr. José António Couto, ESBUC, College of Biotechnology, Portuguese Catholic University, Porto, Portugal, for kindly providing the Saccharomyces cerevisiae strain 3507 from the Portuguese Yeast Cell Culture, Gulbenkian Institute of Science, Lisbon, Portugal. We also thank Professor Rui Fontes for carefully reading this manuscript.

\section{REFERENCES}

1. Van Hoof $V$, De Broe ME. Interpretation and clinical significance of alkaline phosphatase isoenzyme patterns. Crit Rev Clin Lab Sci 1994; 31: 197-293.

2. Millan JL, Fishman WH. Biology of human alkaline phosphatases with special reference to cancer. Crit Rev Clin Lab Sci 1995; 32: 1-39.

3. Martins MJ. Fosfátase alcalina - actividades em diversas situações fisiológicas e patológicas. Relação com sistemas de transporte membranar. [Doctoral thesis]. Porto: Biochemistry Department, University of Porto; 2001.

4. McComb RB, Bowers GN Jr. Study of optimum buffer conditions for measuring alkaline phosphatase activity in human serum. Clin Chem 1972; 18: 97-104.

5. Donella-Deana A, Ostojic S, Pinna LA, Barbaric S. Specific dephosphorylation of phosphopeptides by the yeast alkaline phosphatase encoded by $\mathrm{PHO} 8$ gene. Biochim Biophys Acta 1993; 1177: 221-228.

6. Tuleva B, Vasileva-Tonkova E, Galabova D. A specific alkaline phosphatase from Saccharomyces cerevisiae with protein phosphatase activity. FEMS Microbiol Lett 1998; 161: 139-144.

7. Galabova D, Tuleva B, Vasileva-Tonkova E, Christova N. 
Purification and properties of alkaline phosphatase with protein phosphatase activity from Saccharomyces cerevisiae. Z Naturforsch [C] 2000; 55: 588-593.

8. Song L. A soluble form of phosphatase in Saccharomyces cerevisiae capable of converting farnesyl diphosphate into E,E-farnesol. Appl Biochem Biotechnol 2006; 128: 149-158.

9. Hui M, Tenenbaum HC. New face of an old enzyme: alkaline phosphatase may contribute to human tissue aging by inducing tissue hardening and calcification. Anat Rec 1998; 253: 91-94.

10. Hui M, Li SQ, Holmyard D, Cheng P. Stable transfection of nonosteogenic cell lines with tissue nonspecific alkaline phosphatase enhances mineral deposition both in the presence and absence of beta-glycerophosphate: possible role for alkaline phosphatase in pathological mineralization. Calcif Tissue Int 1997; 60: 467-472.

11. Nishimura K, Yasumura K, Igarashi K, Harashima S, Kakinuma Y. Transcription of some PHO genes in Saccharomyces cerevisiae is regulated by spt7p. Yeast 1999; 15: 1711-1717.

12. Scheibe RJ, Kuehl H, Krautwald S, Meissner JD, Mueller $\mathrm{WH}$. Ecto-alkaline phosphatase activity identified at physiological $\mathrm{pH}$ range on intact $\mathrm{P} 19$ and $\mathrm{HL}-60$ cells is induced by retinoic acid. J Cell Biochem 2000; 76: 420-436.

13. Martins MJ, Negrão MR, Hipolito-Reis C. Alkaline phosphatase from rat liver and kidney is differentially modulated. Clin Biochem 2001; 34: 463-468.

14. Martins MJ, Negrão MR, Hipolito-Reis C, Azevedo I. Physiologic concentrations of bile salts inhibit rat hepatic alkaline phosphatase but not the intestinal isoenzyme. Clin Biochem 2000; 33: 611-617.

15. Calhau C, Martel F, Hipolito-Reis C, Azevedo I. Modulation of uptake of organic cationic drugs in cultured human colon adenocarcinoma Caco-2 cells by an ecto-alkaline phosphatase activity. J Cell Biochem 2002; 87: 408-416.

16. Calhau C, Martel F, Soares-da-Silva P, Hipolito-Reis C, Azevedo I. Regulation of [(3)H]MPP(+) transport by phosphorylation/dephosphorylation pathways in RBE4 cells: role of ecto-alkaline phosphatase. Naunyn Schmiedebergs Arch Pharmacol 2002; 365: 349-356.

17. Calhau C, Martel F, Pinheiro-Silva S, Pinheiro H, Soaresda-Silva P, Hipolito-Reis C, et al. Modulation of insulin transport in rat brain microvessel endothelial cells by an ectophosphatase activity. J Cell Biochem 2002; 84: 389-400.

18. Ciancaglini $P$, Simao AM, Camolezi FL, Millan JL, Pizauro JM. Contribution of matrix vesicles and alkaline phosphatase to ectopic bone formation. Braz J Med Biol Res 2006; 39: 603-610.

19. Mitchell JK, Fonzi WA, Wilkerson J, Opheim DJ. A particulate form of alkaline phosphatase in the yeast, Saccharomyces cerevisiae. Biochim Biophys Acta 1981; 657: 482-494.

20. Klionsky DJ, Emr SD. Membrane protein sorting: biosynthesis, transport and processing of yeast vacuolar alkaline phosphatase. EMBO J 1989; 8: 2241-2250.

21. Hayashi N, Oshima Y. Specific cis-acting sequence for $\mathrm{PHO} 8$ expression interacts with $\mathrm{PHO} 4$ protein, a positive regulatory factor, in Saccharomyces cerevisiae. Mol Cell Biol 1991; 11: 785-794.

22. Spasova D, Galabova D. Yeast permeabilization as a tool for measurement of in situ enzyme activity: localization of alkaline phosphatase. Z Naturforsch [C] 1998; 53: 347-351.

23. Tan I, Moss DW. The estimation of intestinal alkaline phos- phatase in human blood serum. Clin Chim Acta 1969; 25: 117-125.

24. Sakiyama T, Robinson JC, Chou JY. Multiple forms of alkaline phosphatase in untreated and 5-bromo-2'-deoxyuridinetreated choriocarcinoma cells. Arch Biochem Biophys 1978; 191: 782-791.

25. Hummer C, Millan JL. Gly429 is the major determinant of uncompetitive inhibition of human germ cell alkaline phosphatase by L-leucine. Biochem J 1991; 274 (Part 1): 91-95.

26. Mota A, Calhau C, Martel F, Martins MJ. Modulation of rat heart alkaline phosphatase activity by drugs, hormones and nutrients. FASEB J 2006; 20: A897 (Abstract 588.12).

27. Negrão MR, Mota A, Azevedo I, Martins MJ. Statins and tissue mineralization: putative involvement of alkaline phosphatase. Med Hypotheses 2006; 67: 524-528.

28. Negrão MR, Keating E, Faria A, Azevedo I, Martins MJ. Acute effect of tea, wine, beer, and polyphenols on ectoalkaline phosphatase activity in human vascular smooth muscle cells. J Agric Food Chem 2006; 54: 4982-4988.

29. Bradford MM. A rapid and sensitive method for the quantitation of microgram quantities of protein utilizing the principle of protein-dye binding. Anal Biochem 1976; 72: 248-254.

30. Conyers RA, Birkett DJ, Neale FC, Posen S, BrudenellWoods J. The action of EDTA on human alkaline phosphatases. Biochim Biophys Acta 1967; 139: 363-371.

31. Whisnant AR, Gilman SD. Studies of reversible inhibition, irreversible inhibition, and activation of alkaline phosphatase by capillary electrophoresis. Anal Biochem 2002; 307: 226-234.

32. Bhatti AR. Some distinctive characteristics of the alkaline phosphatase of Serratia marcescens. Can J Biochem 1975; 53: 819-822.

33. Mager WH, Winderickx J. Yeast as a model for medical and medicinal research. Trends Pharmacol Sci 2005; 26: 265-273.

34. Feldman D, Tokes LG, Stathis PA, Miller SC, Kurz W, Harvey D. Identification of 17 beta-estradiol as the estrogenic substance in Saccharomyces cerevisiae. Proc Natl Acad Sci USA 1984; 81: 4722-4726.

35. Miller SC, Bottema CD, Stathis PA, Tokes LG, Feldman D. Unexpected presence of estrogens in culture medium supplements: subsequent metabolism by the yeast Sacchromyces cerevisiae. Endocrinology 1986; 119: 1362-1369.

36. Qin YM, Poutanen MH, Novikov DK. Substrate specificities of peroxisomal members of short-chain alcohol dehydrogenase superfamily: expression and characterization of dehydrogenase part of Candida tropicalis multifunctional enzyme. J Lipid Res 2000; 41: 93-98.

37. Motizuki M, Tsurugi K. Effect of 17 beta-estradiol on the generation time of old cells of the yeast Saccharomyces cerevisiae. Biochem Biophys Res Commun 1992; 183: 1191-1196.

38. Plankert $\mathrm{U}$, Purwin $\mathrm{C}$, Holzer $\mathrm{H}$. Yeast fructose-2,6-bisphosphate 6-phosphatase is encoded by $\mathrm{PHO}$, the gene for nonspecific repressible alkaline phosphatase. Eur J Biochem 1991; 196: 191-196.

39. Narisawa S, Huang L, Iwasaki A, Hasegawa H, Alpers DH, Millan JL. Accelerated fat absorption in intestinal alkaline phosphatase knockout mice. Mol Cell Biol 2003; 23: 75257530.

40. Malo MS, Mozumder M, Zhang XB, Biswas S, Chen A, Bai $\mathrm{LC}$, et al. Intestinal alkaline phosphatase gene expression is activated by ZBP-89. Am J Physiol Gastrointest Liver Physiol 2006; 290: G737-G746. 